\title{
A Sphaghetti Sign in the Abdominal Radiograph Consistent with Spleno-Systemic Shunts in a Cat
}

\author{
Donghyun Oh, Jaewoo Hwang, Junghee Yoon and Mincheol Choi ${ }^{1}$ \\ College of Veterinary Medicine and the Research Institute for Veterinary Science, Seoul National University, Seoul 08826, Korea
}

(Received: May 29, 2020 / Accepted: August 11, 2020)

\begin{abstract}
A 8-year-old spayed female Korean short-haired cat was presented with respiratory distress. CBC, serum chemistry analysis, plain radiography, and abdominal ultrasonography were performed. Besides hypertrophic cardiomyopathy (HCM) suspected by the thoracic radiograph, a tubular, tortuous soft tissue structure was detected at the region of the left retroperitoneal cavity on the abdominal radiograph. On the abdominal ultrasonography, a shunt vessel is identified caudo-lateral to the left kidney region. These findings are consistent with spleno-systemic shunts in cats. Furthermore, portal hypertension and diffuse hepatic lesion were also identified. Although the cause of a shunt vessel is not easy to diagnose, it is important to include spleno-systemic shunt into differential diagnosis list, when convoluted, tubular soft tissue opacity is seen on the digital radiography (DR). This report will allow clinicians to raise awareness of complications of portosystemic shunt (PSS) and better treat PSS suspected feline patients when the advanced modalities such as computed tomography and magnetic resonance imaging are not available.
\end{abstract}

Key words : Sphaghetti sign, portosystemic shunt, spleno-systemic shunts, feline, soft tissue opacity.

\section{Introduction}

Portosystemic shunt (PSS) is an anomalous vessel developed from congenital cause or conditioned reasons. In cats, the most common form of PSS is congenital PSS and the extrahepatic form prevails over the intrahepatic form (2-10).

According to the previous study, the crooked tube-like soft tissue structure, also known as "spaghetti sign," visible at the level of left mid-abdomen in cats on DR is suggestive of spleno-systemic collateral circulation (2). Therefore, it is critical if a tubular soft tissue opacity is detected caudo-lateral to the left kidney region, consideration of portal collaterals should be given.

DR is one of the most common and useful medical diagnostic procedures. So it is routinely used when clinicians undergo diagnostic tests of many different patients. The purpose of this report is to present the radiographic feature of spleno-systemic shunts in cats and inform usefulness of commonly encountered modality in practice routine.

\section{Case}

An 8-year-old spayed female Korean short-haired cat was hospitalized emergently with the chief complaint of tachy-

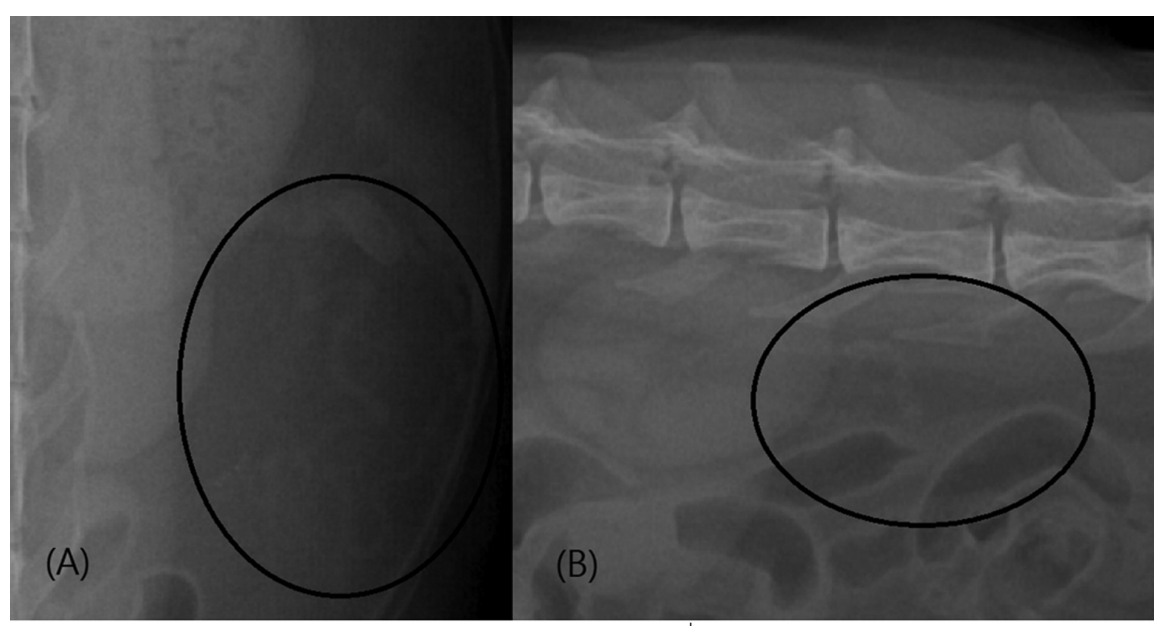

Fig 1. Ventrodorsal (A) and right lateral (B) abdominal radiography. A tortuous, tubular soft tissue opacity is marked with black circles. 
pnea suddenly started in the morning. The cat was diagnosed hypertrophic cardiomyopathy (HCM) by a referring veterinarian and had the history of pemphigus foliaceous diagnosed at Seoul National University Veterinary Medical Teaching Hospital. Vital signs such as temperature, pulse, and respiration was unremarkable at the time of presentation whereas serum chemistry showed significant increases in the liver panel and glucose level. Radiographic examination was followed, in which a twisted tube-shaped soft tissue structure was detected caudo-lateral to the region of the left kidney (Fig 1). The dimension of this structure was not thick enough as intestines nor uterine remnants.

In relation to increased size of heart and pulmonary vessels on radiograph, echocardiography was further performed (Aloka Prosound Alpha- $7^{\mathbb{}}$; Hitachi, Japan). On the examination, along with regurgitant jet flow filling more than $50 \%$ of left atrium, symmetrically increased thickness of left ventricle wall was confirmed, which supported the possibility of

\section{HCM.}

After the echocardiographic assessment, abdominal ultrasonography was performed for comprehensive examination regarding the increase in liver enzyme panel. General anesthesia nor sedation was not induced owing to the cat's favorable manner. It was found that the liver had increased echogenicity of the parenchyma with fine echotexture of the left portion of the liver (Fig 2), and the mean velocity of portal vein was less than $10 \mathrm{~cm} / \mathrm{s}$, indicating portal hypertension. Because of the parabolical movement of the portal flow, the mean portal flow velocity is calculated by multiplying the maximum velocity by 0.57 (27), and in this case, it was $5.7 \mathrm{~cm} / \mathrm{s}$ (Fig 3). Furthermore, aberrant tortuous blood vessels arising from the splenic vein was coursing caudally to the left kidney and continued to the caudal vena cava (CVC) adjacent to the renal vein (Fig 4). These findings are consistent with the spleno-systemic shunt in cats. Although the shunt vessel is not easy to diagnose when clinical signs of

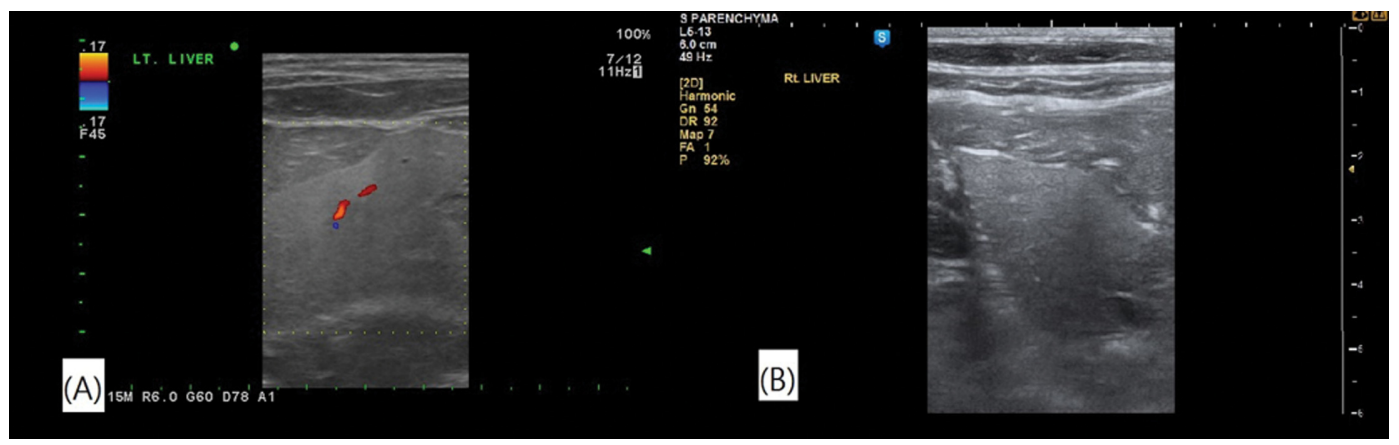

Fig 2. Transverse ultrasonographic images of the left (A) and right liver (B) parenchyma. Note the fine echotexture and increased echogenicity of the hepatic parenchyma.

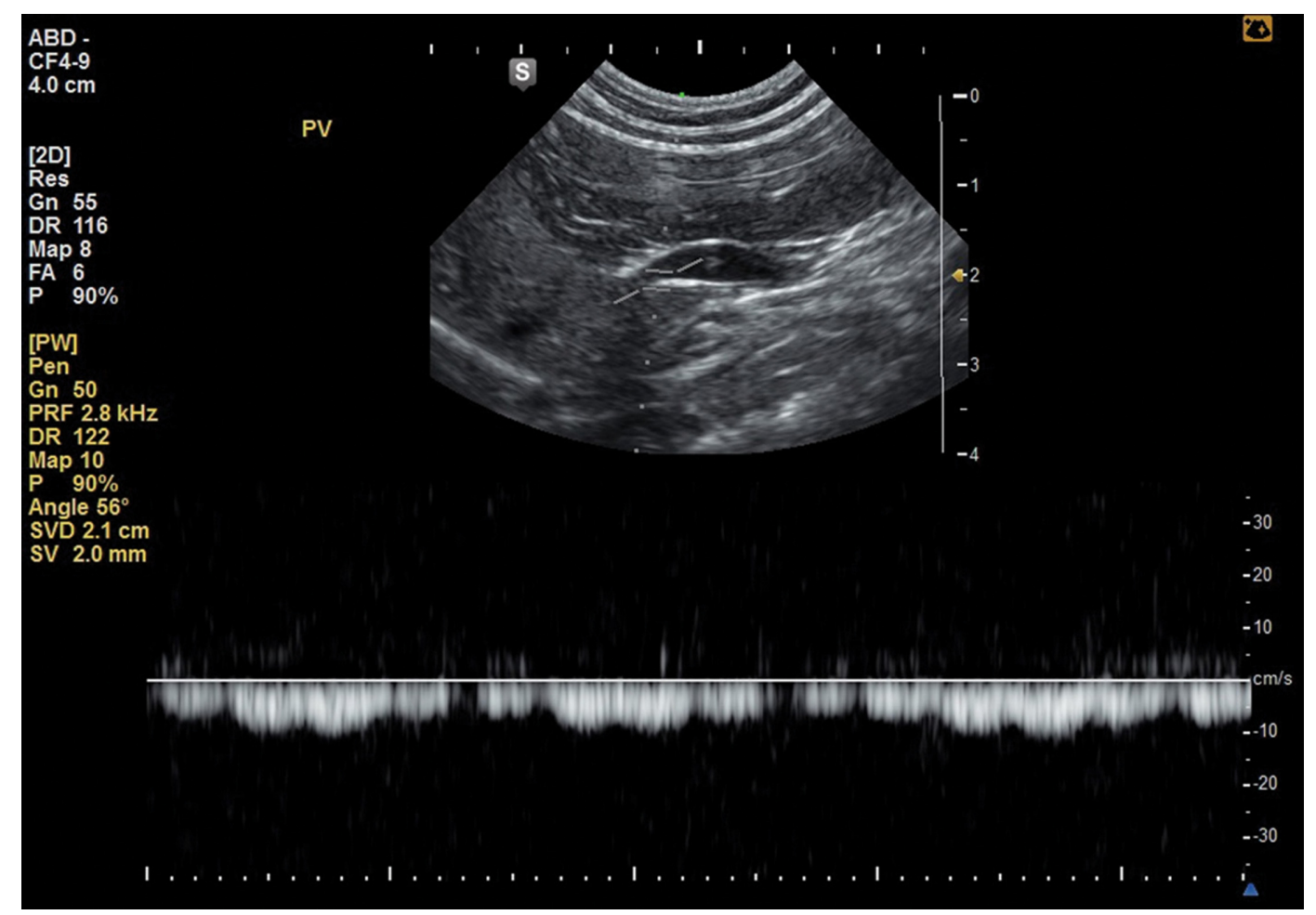

Fig 3. Spectral Doppler pattern of the portal flow. Mean flow velocity is calculated by multiplying the maximal velocity $(10 \mathrm{~cm} / \mathrm{s})$ by a factor of 0.57 , which equals $5.7 \mathrm{~cm} / \mathrm{s}$. The angle of sample volume correction was $56^{\circ}$. 


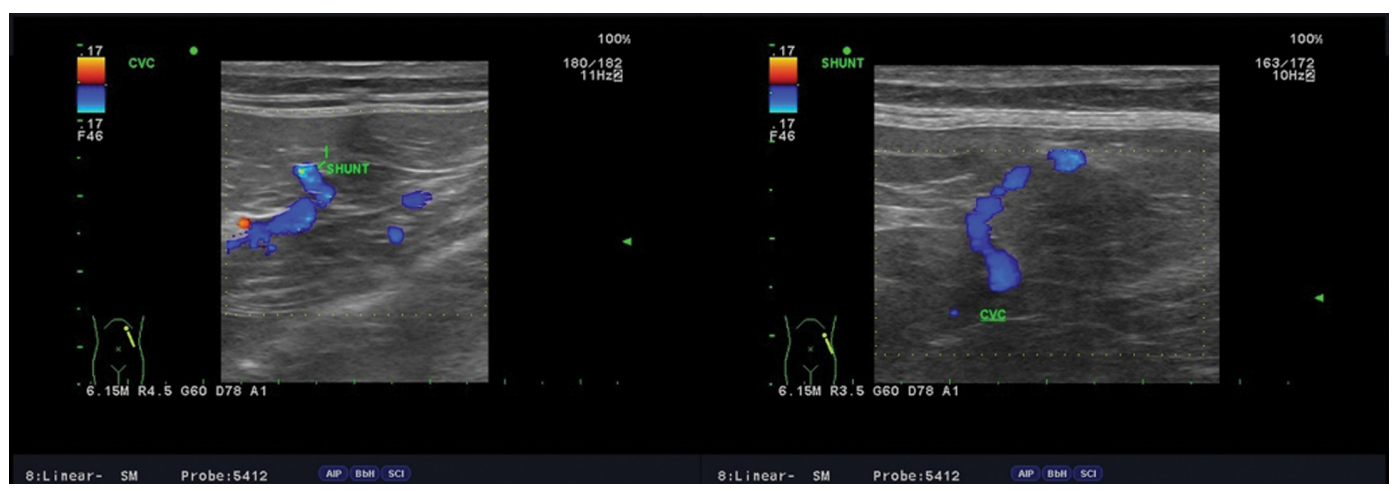

Fig 4. Sagittal ultrasonographic images at the level of region caudal to the left kidney. A shunting vessel was confirmed by color Doppler ultrasound. It was arising from the splenic vein and continued to CVC.

PSS is absent, it seems necessary to include spleno-systemic shunt into differential diagnositic list, when convoluted, tubular soft tissue opacity is seen on radiograph.

\section{Discussion}

Although PSS is not considered when evaluating the patient, they were diagnosed incidentally in this case by detecting a shunt vessel connected to $\mathrm{CVC}$ at the region caudolateral to the kidney through ultrasonography.

Unlike in the cases of canine diseases, PSS is reported less often than those of feline (2,6,11-14). Congenital PSS is not commonly seen in cats due to the challenging recognition of this disease because they may occur without any clinical signs. However, congenital PSS is the most commonly described PSS in cats than acquired PSS is. The patient in this study is considered to be representing acquired type of PSS. According to experimental models of portal hypertension, acquired PSS results from the communication between the portal and systemic circulation by non-degenerated embryonic vessels, leading to diversion of portal blood flow into the systemic circulation (15-18). It was confirmed that conditions such as hepatic fibrosis $(13,19,20)$, arteriovenous fistulas $(21,22)$, portal vein thrombosis or intraluminal masses $(21,23)$, portal vein hypoplasia (24), and attenuation of a congenital PSS have the potential to develop the acquired PSS (19). By evaluating the portal blood flow with pulsed Doppler ultrasound, mean velocity of portal vein below $10 \mathrm{~cm} / \mathrm{s}$ implies the possibility of portal hypertension, which is the most common cause of acquired portosystemic circulation (1-3). Besides shunt vessel, there was an increase in echogenicity at the liver with fine echotexture. Yet, the most commonly occurring clinical signs of congenital PSS such as ptyalism or neurologic signs were not present in this case.

Commonly known, when portosystemic shunts are suspected in domestic animals, abdominal ultrasonography or computed tomography angiography (CTA) scan should be performed to confirm portal collaterals. However, abnormal shunt vessel can be seen on the plain radiograph in the cases of cats (1). Therefore, when there is a twisted tubular soft tissue opacity at the retroperitoneal region caudal to kidney, spleno-systemic shunts should be taken into account regarding the differential diagnosis.

According to the patient's history, the feline patient in this case had long courses of corticosteroids and short courses of immunosuppressants concerning the oral disease and chronic dermal issue. There may be a relationship between the use of such medications and portal hypertension. It is possible that excessive use of iatrogenic corticosteroids can ensue portal hypertension by inducing portal vein thrombosis, but no thrombosis was found by ultrasonographic examination $(25,26)$. There were no significant supporting ideas that immunosuppressants contribute to a decrease in portal blood flow velocity. So, the assumption that the medications could have influenced the portal blood flow is out of the question. On the other hand, the patient was spayed and it is probable that the complication following the ovariohysterectomy could be attributable to neovascularization of collateral vessel (1).

In this case, a few limitations were present. Due to the owner's refusal to further examine the patient with advanced diagnostic method such as CTA, it was not possible to identify the morphology of spleno-systemic collateral circulation PSS. Moreover, it was not feasible to follow up the patient because the owner was not willing to take the treatment at our institution. Whether these PSS was congenital or acquired in origin could not be determined due to the lack of followups. Further investigation is needed to analyze which type of PSS is overrepresented in spayed female cats.

\section{Conclusions}

In conclusion, one of the most frequently used diagnostic tests, plain radiography may provide useful information about abnormal portosystemic circulation in adult cats. Localization of a tortuous tubular structure with soft tissue opacity caudal or lateral to the region of the left kidney is essential because it is indicative of a spleno-systemic shunt vessel. This significant radiographic finding will afford clinicians to take into consideration a series of complications when treating the feline patients with potential PSS when the CTA or MRI is absent.

\section{References}

1. Specchi S, Panopoulos I, Adrian AM, Spattini G, Morabito S, Paek M. A "spaghetti sign" in feline abdominal radiographs predicts spleno-systemic collateral circulation. Vet Radiol Ultrasound 2018; 59: 13-17. 
2. Hunt GB. Effect of breed on anatomy of portosystemic shunts resulting from congenital diseases in dogs and cats: A review of 242 cases. Aust Vet J 2004; 82: 746-749.

3. Levy JK, Bunch SE, Komtebedde J. Feline porto-systemic vascular shunts. In: Bonagura JD, ed. Kirk's current veterinary therapy XII small animal practice. Philadelphia: WB Saunders. 1995: 743-749.

4. Birchard SJ, Sherding RG. Feline portosystemic shunts. Compend Contin Educ Pract Vet 1992; 14: 1295-1301.

5. Lipscomb VJ, Lee KC, Lamb CR, Brockman DJ. Association of mesenteric portovenographic findings with outcome in cats receiving surgical treatment for single congenital portosystemic shunts. J Am Vet Med Assoc 2009: 15; 234: 221-228.

6. Lipscomb VJ, Jones HJ, Brockman DJ. Complications and long-term outcomes of the ligation of congenital portosystemic shunts in 49 cats. Vet Rec 2007; 160: 465-470.

7. Wolschrijn CF, Mahapokai W, Rothuizen J, Meyer HP, van Sluijs FJ. Gauged attenuation of congenital portosystemic shunts: results in 160 dogs and 15 cats. Vet Quart 2000; 22: 94-98.

8. White RN, Macdonald NJ, Burton CA. Use of intraoperative mesenteric portovenography in congenital portosystemic shunt surgery. Vet Radiol Ultrasound 2003; 44: 514-521.

9. Van den Ingh TSGAM, Rothuizen J, Meyer HP. Circulatory disorders of the liver in dogs and cats. Vet Quart 1995; 17: 70-76.

10. Kyles AE, Hardie EM, Mehl M, Gregory CR. Evaluation of ameroid ring constrictors for the management of single extrahepatic portosystemic shunts in cats: 23 cases (19962001). J Am Vet Med Assoc 2002; 220: 1341-1347.

11. Cabassu J, Seim HB, MacPhail CM, Monnet E. Outcomes of cats undergoing surgical attenuation of congenital extrahepatic portosystemic shunts through cellophane banding: 9 cases (2000-2007). J Am Vet Med Assoc 2011; 238: 89-93.

12. Hunt GB, Kummeling A, Tisdall PLC, Marchevsky AM, Liptak JM, Youmans KR, Goldsmid SE, Beck JA. Outcomes of cellophane banding for congenital portosystemic shunts in 106 dogs and 5 cats. Vet Surg 2004; 33: 25-31.

13. Langdon P, Cohn LA, Kreeger JM, Priddy NH. Acquired portosystemic shunting in two cats. J Am Anim Hosp Assoc 2002; 38: 21-27.

14. Tillson DM, Winkler JT. Diagnosis and treatment of porto- systemic shunts in the cat. Vet Clin Small Anim 2002; 32: 881-899.

15. Howe LM, Boothe HW Jr, Miller MW, Boothe DM. A canine model of multiple portosystemic shunting. J Invest Surg 2000; 13: 45-57.

16. Bosch J, Enriquez R, Groszmann RJ, Storer EH. Chronic bile duct ligation in the dog: Hemodynamic characterization of a portal hypertensive model. Hepatology 1983; 3: 1002-1007.

17. Ingles AC, Legare DJ, Lautt WW. Development of portacaval shunts in portal-stenotic cats. Can J Physiol Pharmacol 1993; 71: 671-674.

18. Khan IR, Vitums A. Portosystemic communications in the cat. Res Vet Sci 1971; 12: 215-218.

19. Zandvliet MM, Szatmari V, van den Ingh T, Rothuizen J. Acquired portosystemic shunting in 2 cats secondary to congenital hepatic fibrosis. J Vet Intern Med 2005; 19: 765767.

20. Gores BR, Berg J, Carpenter JL, Ullman SL. Chylous ascites in cats: Nine cases (1978-1993). J Am Vet Med Assoc 1994; 205: 1161-1164.

21. McConnell JF, Sparkes AH, Ladlow J, Doust R, Davies S. Ultrasonographic diagnosis of unusual portal vascular abnormalities in two cats. J Small Anim Pract 2006; 47: 338-343.

22. Legendre AM, Krahwinkel DJ, Carrig CB, Michel RL. Ascites associated with intrahepatic arteriovenous fistula in a cat. J Am Vet Med Assoc 1976; 168: 589-591

23. Rogers CL, O'Toole TE, Keating JH, Penninck DG, Webster CRL. Portal vein thrombois in cats: 6 cases (2001-2006). J Vet Intern Med 2008; 22: 282-287.

24. Hawe RS, Mullen HS. An unusual portacaval anomaly as a cause of hepatic encephalopathy in a cat. J Am Anim Hosp Assoc 1984; 20: 987-993.

25. Díaz-Espiñeira MM, Vink-Nooteboom M, van den Ingh TS, Rothuizen J. Thrombosis of the portal vein in a miniature schnauzer. J Small Anim Pract 1999; 40: 540-543.

26. Laurenson MP, Hopper K, Herrera MA, Johnson EG. Concurrent diseases and condition in dogs with splenic vein thrombosis. J Vet Intern Med 2010; 24: 1298-1304.

27. Nyland TG, Fisher PE. Evaluation of experimentally induced canine hepatic cirrhosis using duplex Doppler ultrasound. Vet Radiol 1990; 31: 189-194. 